\title{
Research on Information Security of Digital Library under the Cloud Computing Environment
}

\author{
Yun Lei \\ Library, Jiangxi University of Science and Technology, Ganzhou, 341000, China
}

Keywords: Digital library; Cloud computing; Information security.

\begin{abstract}
Cloud computing model as the information system of modern digital library operation infrastructure, it in the data sharing, application platform, software environment and so on the continuous optimization of the library reform, improve the library service, deeply affect the operation and service mode of digital library type. This paper introduces the concept and characteristics of cloud computing, analysis the relationship between cloud computing and digital library, the summary of cloud computing security management problems under the environment of digital libraries, discussion of cloud computing in information security management measures under the environment of digital libraries, to adopt the cloud model optimized libraries, improve service level and promoting the library.
\end{abstract}

\section{General instructions}

New service computing model of "cloud computing" as a new application mode, can connect many servers, give full play to the super computing power required by the server, in order to achieve more user groups to create more high-quality service projects and the use of performance goals. Cloud computing into the digital library, not only can provide large servers and mass storage devices such as the hardware core is the digital library in the framework of the hardware level, data computing operating system and can provide digital library management at the level of software and software environment. Combined with cloud computing technology and digital library and realize the information resource scale and centralized, to build a digital library complete cloud computing platform.

\section{Cloud Computing}

\subsection{The definition and characteristics of cloud computing}

Cloud computing is a distributed computing technology is the development of processing, parallel processing, grid computing, computer science concepts to achieve commercial. The core of cloud computing is computing and storage of mass data, it adopted the huge computational processing program split into numerous smaller subroutines, and then by the linked server group to search and calculation, analysis and processing results will return to the user, so that the computing power as a kind of commodity circulation.

Cloud computing to the user as the center, through the network to provide users with safe, convenient data storage function and network service. Cloud computing is mainly has the following four characteristics:

1) Safe and reliable data storage function. Cloud computing environment can make real-time synchronous data transmission, and can pass the Web on the terminal equipment use, thus exclude users worry about data stored in the personal computer and the emergence of lost or infected concerns.

2) Convenient network service function. The cloud server for IT professionals can help users to maintain the hardware facilities, install the upgrade software, to prevent network attacks, so that users don't need to unlimited upgrade their operating system and computer hardware, do not need to repeatedly install firewall and antivirus software, terminal only needs to have a web browser can enjoy fast and convenient network service. 
3) The powerful data calculation processing function. Cloud computing is composed of hundreds of thousands of computers co integration operation, store huge amounts of data, provide the powerful computing ability, complete the general computer is difficult to complete the business.

4) Cloud computing easy access, sharing and strong. Cloud computing network application mode of data stored in the cloud server, the user can will cloud personal operating system access, data sharing and document and other operating system. Therefore, the user can query to more information in different databases.

\subsection{Cloud computing and digital library}

In recent years, many libraries were digitized processing large, not only make the library information resources have been accumulated, but also make the data technology has been constantly updated and development. The birth of cloud computing for the digital library provides users with better service function; the key is to enhance the digital library's service mode and efficiency. The users of Digital Library under the cloud computing environment can not only buy the software right to use, also can buy virtual application software services directly to the "cloud". Cloud computing system is a virtual pool of resources, digital library to provide through the Internet to buy cloud service providers IT outsourcing management services, in order to ensure the digital library users ready access to cloud platform and get instant information retrieval.

\section{Information security management problems of Digital Library under the cloud computing environment}

First of all, "the cloud" the user's dynamic, service diversity and computational complexity are affecting the digital library information resources security, data integrity and confidentiality. Because cloud computing is a complex huge system, it is transmitting, massive data processing and storage process will cause the destruction of data information, stolen or lost. However, cloud computing providers only spread in the network information encryption to protect data, so the digital library data storage security become the core problem of Digital Library under the cloud computing environment which need to be solved.

Secondly, due to different users of Digital Libraries in cloud computing needs, personal data may be scattered in different virtual data center, when the host is attacked, access to virtual machine client server may also be overcome, then lead to the invasion of illegal users of cloud computing to steal confidential data users of digital library system environment, integrity and confidentiality of serious the threat of library user data. Multimedia digital resources of library management, library users' personal database once the data is lost or stolen, and the consequences will be unbearable to contemplate. How to avoid the potential risk of coexisting group service for library users, has become an important challenge for cloud computing security management of digital library environment.

\section{Cloud computing, information security management measures under the environment of Digital Libraries}

Computational requirements of information security management under the environment of Digital Libraries in the cloud, digital library should be based on the new technology of the current development situation, take measures of safety management and reasonable construction of "Library of cloud", to provide a stable, safe, reliable calculation ability of super network platform for the digital library legal user group.

\subsection{Using the virtual mass storage technology, ensure the security of data storage of Digital Library}

In order to cloud computing is no danger of anything going wrong data under the environment of digital library, service providers mainly uses the virtual mass storage technology of advanced management and storage of data resources. The virtual mass storage technology is commonly used image and calibration technology of fault tolerance, rely on two different sets of equipment to maintain the same data information, if the master device is damaged, you can switch to the mirror to 
access equipment. The virtual mass storage technology by using this data copy way fault tolerance does not need redundant equipment, only the hardware requirements of the digital library reasonable equipment configuration and placed reasonable, effectively avoid or reduce the risk of hardware equipment destroyed. First, the digital library hardware device to install the best in a special room, to ensure that the resettlement point is far away from flammable and explosive places. Second, belonging to the digital library system equipment center buildings must have the ability to resist natural disasters, and the need for the establishment of a multilayered security protection ring in the device center periphery prevent the intrusion of illegal violence. At the same time, also need to strengthen fire prevention, waterproof, anti-theft measures, regular maintenance of hardware equipment. In addition to cloud virtualization storage technology, the digital library should also be on the data resources of each collection self-comprehensive and timely backup and save the long-term reliable.

\subsection{Unified user identity authentication of digital libraries, to ensure the security of cloud computing platform}

The unified authentication of user identity information is a prerequisite to achieve data and service security of digital library, especially the safety management under the environment of digital libraries is essential in cloud computing. Because cloud computing environment with dynamic, heterogeneous and cross organization, to require all cloud resources for identity authentication user group. Therefore, the Digital Library under the cloud computing environment requires users using single point login for the identity information unified authentication, only by authorized user authentication before they can enjoy the cloud resources. As long as, once the library user identity is authorized by the certification, access to other authorized cloud resources, which can not only enhance the efficiency of access users, but also through the reasonable access control to ensure security of the cloud platform in satisfying the open digital library at the same time.

\subsection{Strengthen the construction of information security infrastructure, to meet the demand of data security in Digital Library}

Public key infrastructure (PKI) by public key cryptography technology, digital certificate, certificate authentication center and on the basic components of public key security policy. System or platform provides and key and certificate management service. Public key infrastructure construction is the core of digital library information security infrastructure, it can make cloud computing digital library environment using the key and the digital signature technology, implementation of cloud data resources are not peek, not to be denied and be non-tampered purposes in transmission and storage process, and meets the requirements of data security of digital library, guarantee the cloud resources confidentiality, integrity and availability.

\subsection{To formulate and improve relevant policies and regulations, safeguard the legitimate rights and interests of the digital library}

Because cloud computing digital library under the environment of data security and privacy problems will involve the issue of protection of intellectual property rights, responsibility for the rights and interests of all parties, especially relates to the policy level problem, have certain negative impact of these on cloud computing data security. Therefore, the digital library industry managers need to calculate the "service provider" Research on evaluation index system of cloud strengthen, to explore the related industry standards for cloud computing applications, formulate and perfect the reasonable management regulations and policies and regulations system, the effective management of cloud computing application, safeguard the legitimate rights and interests of the Digital Library.

\section{Conclusions}

To sum up, the cloud computing technology, which can not only improve the services of digital library, and optimize the service function of the digital library, but also the safety management for the digital library brings challenges. Digital Library under the cloud computing environment will be facing the involved data storage security problem, the user information privacy and personal rights management problem, cloud data resources rights, these are for the digital library has caused serious 
security hidden danger. Therefore, libraries need to ensure safe use of library data storage virtualization technology of massive information storage cloud data copy fault-tolerant, need unified authentication user identity of access control in order to ensure the cloud platform security, the need to strengthen the construction of information security infrastructure to meet the digital library data security requirements, also need to develop and perfect the relevant policies and regulations to protect the digital library the legitimate rights and interests, and eventually realize the cloud computing under the environment of digital library information resources security, focused and effective.

\section{Reference}

[1] Fan Bingsi. Cloud computing and Library: cloud computing research defense [J]. library and information work of.2009 (53 - 21).

[2] Huang Xingliang, Li Shujun. Research on the study of [J]. Library of mass storage technology in digital library, 2010,12 (9).

[3] Hu Xiaojing, Fan Bingsi. Cloud computing challenges [J]. Journal of university library to library management, 2009 (4): 7-12.

[4] Michael Armbrust, Armando Fox, Rean Griffith, et al. Above the clouds: A Berkeley view of cloud computing [J].Communication Magazine, 2009.

[5] Sun Tan, Huang Guobin. Construction of [J]. library con-struction and service strategy of Library Based on cloud ser-vice, 2009 (9): 1-6.

[6] Tang Zhengjun, Li Jianhua [M]. intrusion detection technol-ogy. Beijing: Tsinghua University press,.2004 (4).

[7] Wang Changquan, AI atmosphere. The digital library infor-mation security issues in cloud computing era [J]. library con-struction, 2010 (1): 50-52.

[8] Zhang Haiyu. Study on security strategy research, learning [J]. Library of Digital Library under the cloud platform, 2013, (3):42 44. 